\title{
STRUCTURE OF LAMINAR COFLOW SPRAY FLAMES AT DIFFERENT PRESSURES
}

\author{
STEFANO RUSSO AND ALESSANDRO GOMEZ \\ Yale Center for Combustion Studies \\ Department of Mechanical Engineering \\ Yale University \\ New Haven, CT 06520-8286, USA
}

\begin{abstract}
Experiments were conducted on laminar spray diffusion flames of ethanol/argon burning in oxygen at pressures of 1 and $3 \mathrm{~atm}$. The flames were physically characterized by measuring droplet velocities and sizes by phase-Doppler anemometry, and gas temperature by thin-filament pyrometry and thermocouples. The flames exhibited a cold core, where little vaporization occurred, surrounded by a primary diffusion flame enveloping most of the droplet cloud. Their structure was strongly influenced by the Peclet number for heat transfer that, by regulating the heat penetrating into the core, and, consequently, the growth of the thermal boundary layer, affected the droplet vaporization history. At pressures above the atmospheric, because of density effects on thermal diffusivity, the boundary layer growth above the burner was reduced and so was the vaporization region. Complete evaporation of the droplets before they reached the primary diffusion flame was ensured if a suitably defined Damköhler number of evaporation, $D a_{\mathrm{v}}$, was smaller than 1. Conversely, if $D a_{\mathrm{v}}>1$, droplets penetrated the flame, ignited by a flame transfer process that was captured photographically, and burned isolated on the oxidizer side. These conditions of internal or partial group combustion prevailed in the lower part of the flame. Farther up in the flame, this combustion regime progressively shifted toward that of external group combustion, with fewer and fewer direct droplet/flame interactions.
\end{abstract}

\section{Introduction}

Liquid fuels are the primary energy source in a wide range of applications including industrial and residential furnaces, internal combustion engines, and propulsion systems. In many of these devices, the gas in the combusting chamber is at pressures well above the atmospheric and, in some cases, it can reach conditions that are supercritical for the fuel. Because of their inherent complexities, practical spray combustion systems do not provide a wellcontrolled environment from which the effects of individual variables can be extracted. To parallel developments in purely gaseous environments, attention needs to be focused first on laminar spray flames that are best suited to bridge the gap between classical single-droplet burning studies and practical spray flames. Counterflow laminar flow configurations are simple and can be easily modeled numerically [1-4]. Coflow configurations, on the other hand, are well suited to study group combustion phenomena [5-7]. Under typical conditions of moderate slip between the gaseous phase and the droplets, droplets tend to evaporate as a group surrounded by a primary diffusion flame, in a sort of pseudo-gaseous scenario [6]. Under certain conditions, even direct droplet/flame interaction may occur in which case droplets "punch through" this primary diffusion flame and burn isolated on the oxidizer side. They eventually extinguish when they reach a critical diameter dependent on the dropletgas relative speed [8]. Experimental evidence of direct droplet/flame interaction was presented in Ref. [1].

This work aims at examining laminar spray flames at pressures moderately above the atmospheric. A hint of the inherent difficulties in establishing wellcontrolled laminar flames of these types comes from the realization that developments in this area, even at atmospheric pressure, are fairly recent, dating back no more 10 years. The difficulties are exacerbated at high pressures, with safety issues posed by the large amounts of unburned fuel escaping combustion in the counterflow configuration, and with the intensification at high pressure of the effects of the inevitable buoyancy instabilities in the coflow configuration. This investigation focuses on the structure of coflow laminar spray diffusion flames and how it is affected by a 3 -fold change in pressure. Previous numerical work on these flows highlighted the presence of relatively large droplets that penetrate the flame [7]. However, depending on the timetemperature pathway, even relatively small droplets might escape the reacting layer [9] with resulting emission of unburned hydrocarbons. We will show evidence of this phenomenon and couple it with the 


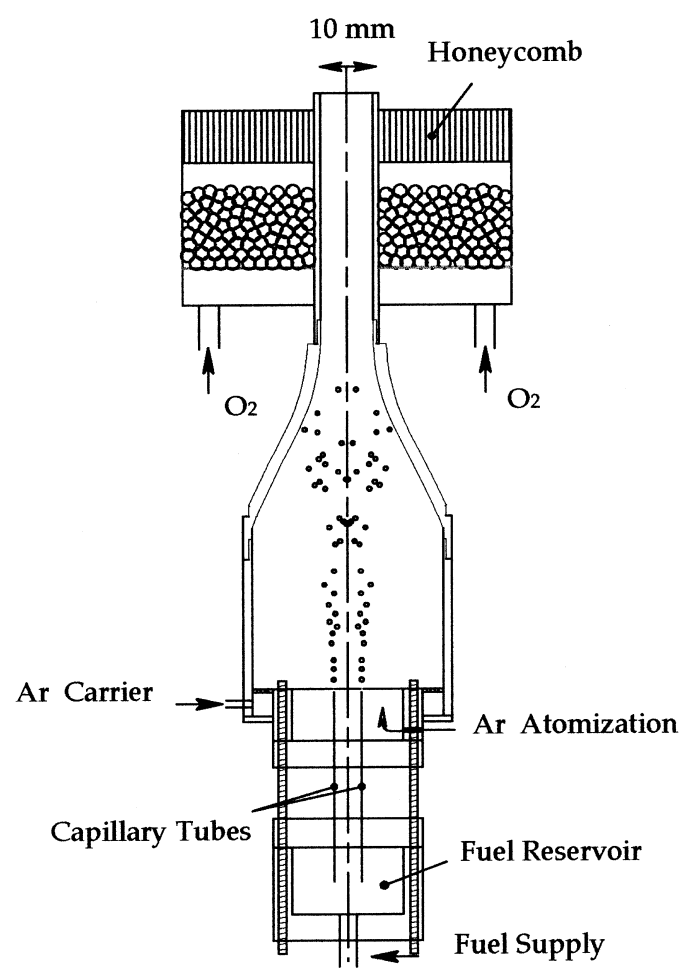

FIG. 1. Burner schematic.

examination of the region in which group combustion prevails.

\section{Experimental Arrangement}

The choice of the atomizer was based on two main considerations: first, the atomization process has to be gentle enough not to disturb the laminarity of the flow; second, maintaining some control of the droplet size distribution would greatly simplify the interpretation of the results.

Pressure atomizers [2] are not well suited since their adaptation to laminar spray flames requires sufficient time for the two phases to equilibrate in a large settling chamber. This would create new difficulties at high pressure and would require large combustion chambers [10]. Ultrasonic atomizers $[3,11]$ are also not appropriate since the pressure waves generated during the atomization process cause strong disturbances to the flame at pressures above $1 \mathrm{~atm}$.

A candidate spray system that satisfies all the necessary prerequisites is a spray generator that we had to develop in house, based on the laminar aerodynamic shearing of a liquid jet by a gaseous coflow [12]. Because of the relatively high flowrate requirements, the atomizer had to be multiplexed. Three parallel metal capillaries were used for the $1 \mathrm{~atm}$ flame and nine for the $3 \mathrm{~atm}$ flame.

This nebulizer was retrofitted to the burner as depicted in Fig. 1. The argon carrier gas entered the duct on the side of the atomizer and carried the spray upward through a contraction terminating in a $7.5 \mathrm{~cm}$ long tube with an inner diameter of $10 \mathrm{~mm}$. Liquid fuel from droplets that impinged on the walls was collected at the base of the duct. The carrier gas bubbled through it and carried it up in the form of vapor. As a result, no significant accumulation of liquid was observed during the experiments.

At the exit of the duct, the droplet-laden jet met with the oxygen stream and burned in a coflow diffusion flame. A system of screens, packed glass beads, and honeycomb guaranteed the laminarity of the oxidizer coflow. The oxygen velocity was kept constant at $\approx 4.5 \mathrm{~cm} / \mathrm{s}$.

Two different flame conditions were investigated. Flame 1, in which $0.7 \mathrm{ml} / \mathrm{min}$ of ethanol were injected into 2.7 standard liters per minute (slpm) of argon at atmospheric pressure, and flame 3, with $2.1 \mathrm{ml} / \mathrm{min}$ of ethanol and $8.1 \mathrm{slpm}$ of argon, at $3 \mathrm{~atm}$. To ensure the attachment of the flame at the tip of the fuel tube some methane had to be added to the fuel side. The methane provided $4 \%$ and $8 \%$ of the total enthalpy for flames 1 and 3, respectively. The liquid fuel was fed into the atomizer from a tank pressurized by nitrogen. Liquid and gas were kept apart in the vessel by a piston to avoid the dissolution of gas into the liquid.

The burner was enclosed in a chamber for highpressure measurements. Two circular glass windows with axis at $65^{\circ}$ provided the optical access for laser light-scattering measurements. The total volume of the chamber was approximately 3 orders of magnitude larger than the inner volume of the burner to avoid buoyancy-induced fluctuations of the fuel and inert mixture flowrate [10]. Because of the limited optical accessibility of the chamber, only the first $20 \mathrm{~mm}$ of the two flames were scanned.

Distributions of droplet size, together with axial and radial velocity components, were measured by a commercial two-velocity component Phase Doppler Anemometer (Dantec Electronik). Gaseous temperatures between $900 \mathrm{~K}$ and $2200 \mathrm{~K}$ were measured using Silica-Carbide thin-filament pyrometry [13]. The signal was collected by an infrared camera (Electrophysics Corp. PV320) with a $50 \mathrm{~mm}$ Germanium lens. During the temperature measurements, the fused silica window of the high-pressure chamber were substituted with an infrared transparent Zinc-Selenide window.

The amount of fuel in the vapor phase at the exit of the burner was measured using two different $\mathrm{K}$ type thermocouples of $3 \mathrm{~mm}$ and $25 \mu \mathrm{m}$ size, respectively. The first thermocouple measured the wet-bulb temperature of the vaporizing flow, while the second one measured the gas temperature. The fuel concentration in the vapor phase can now be 


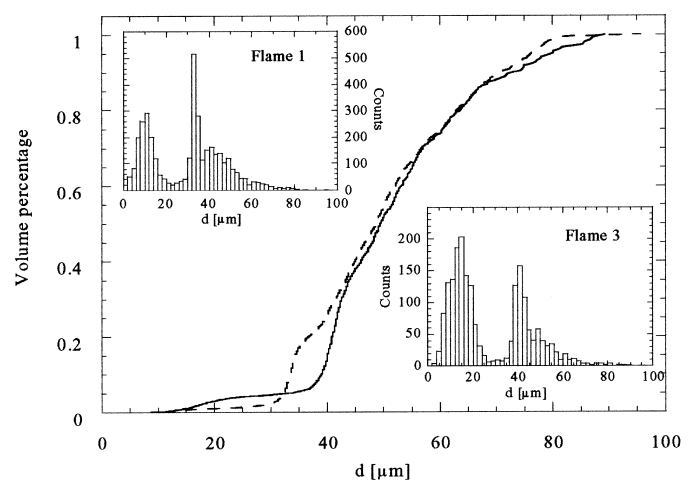

FIG. 2. Typical cumulative volume distribution. Flame 1, dashed line; flame 3, solid line. Insets: droplet size distributions for the different flames.

obtained by equating the B number for heat and mass transfer assuming Lewis number of 1 and pure ethanol in the liquid phase [14].

For the $1 \mathrm{~atm}$ flame, the fuel concentration was calculated this value was $22 \%$ of the total ethanol supplied by the atomizer. The technique was not applicable at higher pressures.

\section{Results and Discussion}

In the atmospheric pressure case, the flame appeared as a steady blue reacting layer measuring approximately $45 \mathrm{~mm}$ in height. The main difference with highly diluted gaseous diffusion flames could be observed near the base. Near the anchoring point and up to approximately 8-10 $\mathrm{mm}$, intermittent luminous streaks were visible, representing the halo of droplets that survived the flame and burned on the outside in the single droplet mode. Higher up in the flame, fewer droplets survived outside the primary diffusion flame. No yellow emission from soot was recorded in any of the two cases.

Differently from flame 1, flame 3 presented a region of buoyancy-driven instabilities in the reacting layer. Its major visible effect was the flickering instability of the top part of the flame $[15,16]$. These fluctuations did not seem to have a significant effect on the lower part of the flame. The maximum amplitude of the axial oscillation was reached at approximately $45-50 \mathrm{~mm}$ well above the part under consideration for this study. At $20 \mathrm{~mm}$, the flame oscillated radially with a $12 \mathrm{~Hz}$ frequency $\pm 0.25 \mathrm{~mm}$ around its average diameter of $9.60 \mathrm{~mm}$ as recorded by temperature measurements. Also, for the high-pressure flame, luminous streaks were visible near the burner mouth. The frequency of droplets exiting the flame also in this case decreased with increasing axial coordinate.

The complete physical characterization of a spray flame requires the measurement of droplet and gas velocity as well as the gas temperature and the droplet size distribution in every point of the flame. Typical distributions of droplet sizes at the exit of the burner for flames 1 and 3 are shown in the insets in Fig. 2. The distribution, because of the high liquid flowrate, is in both cases far from monodisperse, despite claims to the contrary for this kind of atomizer [12]. In the flame 1 distribution, a strong peak around $33 \mu \mathrm{m}$ is clearly visible corresponding to the main mode of Rayleigh breakup of the liquid filament. A smaller peak at about $40 \mu \mathrm{m}$ corresponds to the second harmonic. Other modes of oscillation are buried in the remaining part of the distribution. Clearly visible are also satellite droplets produced during the atomization process and falling in the 0 $20 \mu \mathrm{m}$ range. Similar features are present also in the distribution of the high-pressure flame, for which the main peak is around $40 \mu \mathrm{m}$ and the second harmonic is at $50 \mu \mathrm{m}$. The good correspondence between the two distributions is shown by the main part of Fig. 2 where the volume distribution is compared. The dashed line relates to flame 1 and the continuous line to flame 3 . The two curves are almost superimposed with small differences only in the 33-40 $\mu \mathrm{m}$ range.

The droplet velocity is affected by the gas speed through a balance of drag and inertia. A simple criterion to understand if the droplets follow closely the host fluid is based on the Stokes number that for low droplet Reynolds number can be defined as $S t k=$ $\left(t_{\mathrm{p}} / t_{\mathrm{f}}\right)=\left(\rho_{\mathrm{p}} d_{\mathrm{p}}^{2} U_{\text {avg }} / 18 \mu D\right)$, where the subscript $\mathrm{p}$ refers to particle properties, $\mu$ is the host fluid viscosity, and $t_{\mathrm{f}}=\left(D / U_{\text {avg }}\right)$ is an average fluid time calculated by using the average fluid velocity and the burner diameter. In the present conditions, droplets below $10 \mu \mathrm{m}$ in diameter have a Stokes number lower than $2 \times 10^{-2}$ and can be considered good trackers of the gas.

Axial and radial "gaseous" velocity components are reported in Fig. 3 for the first $20 \mathrm{~mm}$ of the two flames. The left part pertains to flame 3 , whereas the right part represents the atmospheric pressure case. Even in the region of highest radial velocity gradient, the Stokes number, calculated using this gradient as the inverse fluid time, did not reach $7 \times 10^{-2}$. Closest to the exit of the tube, flame $1\left(R e_{1} \approx 300\right)$ has an almost parabolic axial velocity profile with a "bump" on the side in correspondence to the hightemperature region induced by the flame. At higher axial locations, the region influenced by the flame expands toward the center, as required by the growth of the mixing layer between fuel and oxidizer. In this region, the velocity increases because of density changes and buoyancy.

Because of the increased Reynolds number $\left(\mathrm{Re}_{3}\right.$ $\approx 900$ ), flame 3 at the exit of the tube had a relatively flatter profile. Still visible are the flame influence region and the downstream increase in velocity along the wings. The radial velocity presents a region of 


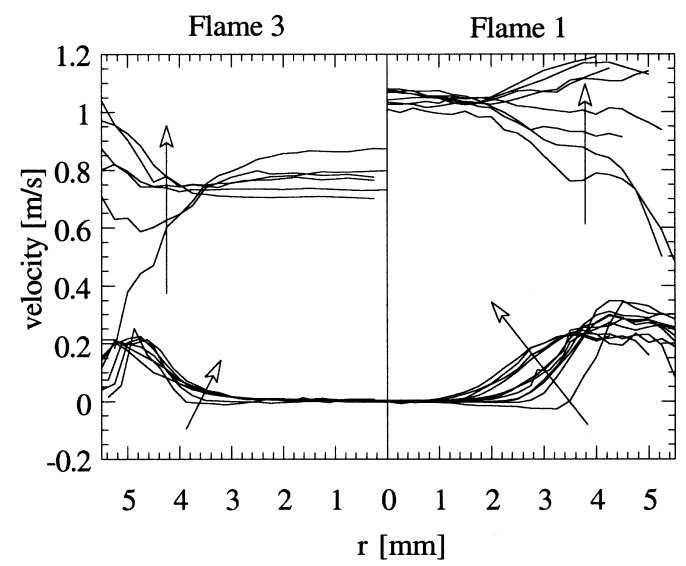

Fig. 3. Axial (top) and radial (bottom) gas-phase velocity component for the two flames as a function of the radial coordinate. The left half pertains to flame 3 (at $z=0.75$, $4,5,8,12,16,18,20 \mathrm{~mm}$ ), the right half to flame 1 (at $z=0.75,2,4,7,10,12,16,18,19.5 \mathrm{~mm}$ ). The arrows in each flame indicate the direction of increasing axial coordinate.

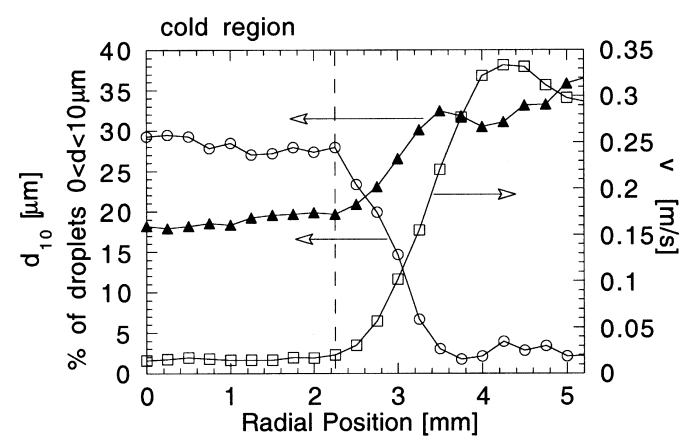

FIG. 4. Left ordinate: average droplet size (triangles) and percentage of droplets in the $0-10 \mu \mathrm{m}$ range (circles) as a function of the radial position. Right ordinate: gas radial velocity component. Measurements pertain to flame 1 at fixed $z=7 \mathrm{~mm}$.

constant, almost zero velocity and external wings with gradient different from zero.

In Fig. 4, the average droplet distribution diameter together with the percentage of droplets below $10 \mu \mathrm{m}$ are plotted as a function of the radial coordinate for the $1 \mathrm{~atm}$ flame at a fixed height $z=7 \mathrm{~mm}$ (left ordinate). The gaseous radial velocity is also reported in the same figure (right ordinate). Interestingly enough, the region of zero radial velocity gradient coincides exactly with the region in which the average diameter and the number percentage stay constant. The constancy of the three properties in the internal part of the flame reveals the presence of a cold core, not affected yet by the heat generated by the flame and in which little or no vaporization takes place. The evolution of the cold core can be followed by any of the three variables reported in Fig. 4. It is controlled by a Peclet number for heat transfer, $P e_{\mathrm{h}}(z)=\left(U_{\mathrm{avg}} z / \alpha\right)$, representing the ratio between axial convective speed and radial diffusive velocity. Because of the density effect on diffusivity, the Peclet number increases linearly with pressure. As a result, at the same axial coordinate, $\left(\mathrm{Pe}_{\mathrm{h} 3}(z)\right) /$ $\left(P e_{\mathrm{h} 1}(z)\right)=3$. For both flames, the cold core width $w$ decreases along the axial coordinate depending on the value of $P e_{\mathrm{h}}$. It should be noted that the initial width $w_{0}$ for the $1 \mathrm{~atm}$ flame is $6 \mathrm{~mm}$, whereas $w_{0}=7.75 \mathrm{~mm}$ for flame 3. As shown in Fig. 3 by the radial velocity, because of the combined effect of $w_{0}$ and $P e_{\mathrm{h}}$, the cold region in flame 3 extends much further in the radial direction than in flame 1.

The positive value of the radial velocity is a typical feature of highly diluted diffusion flames burning in oxygen [17]. In the high-temperature region of a diffusion flame, in fact, two mechanisms tend to accelerate the flow, buoyancy, and thermal expansion. Whereas typical fuel/air coflow diffusion flames are buoyancy dominated [18] and have converging streamlines inside the flame, the present flames are momentum controlled. Thermal expansion is the major mechanism of fuel acceleration and the streamlines diverge.

Once the velocity at different axial positions between 1 and $20 \mathrm{~mm}$ is known, the complete gaseous velocity field can be reconstructed by linear interpolation. Droplets with Stokes number larger than 0.1 will have a small slip with respect to the gas phase and follow different trajectories. By sorting out the droplet velocity on the basis of droplet size, we can reconstruct these trajectories for particles of different size. Seven size classes were used. The first six classes are $10 \mu \mathrm{m}$ wide and range between 0 and $60 \mu \mathrm{m}$, the last one includes all the droplets above $60 \mu \mathrm{m}$. Because of vaporization, a droplet trajectory can be reconstructed only if the complete size-velocity history is known. Thanks to the peculiarity of our distribution, sizes corresponding to the first and second harmonic of flame 1 and to the first harmonic of flame 2 can be experimentally followed along their path.

The evolution of the diameter of a single droplet vaporizing in a quiescent atmosphere can be followed by tentatively applying the $d^{2}$ law, that is,

$$
-\frac{\mathrm{d} d^{2}}{\mathrm{~d} t}=-\frac{8 \lambda}{\rho_{\mathrm{l}} C_{\mathrm{p}}} \ln \left[1+\frac{C_{\mathrm{p}}\left(T_{\infty}-T_{\mathrm{w}}\right)}{L}\right]=K
$$

By assuming argon as the only component and with a suitable choice of the droplet wall temperature $T_{\mathrm{w}}$, the evaporation coefficient reduces to a mere function of the gas-phase temperature, $K(T)$. Once the gas-phase temperature is known, we can use equation 1 to follow the droplet size evolution in the 


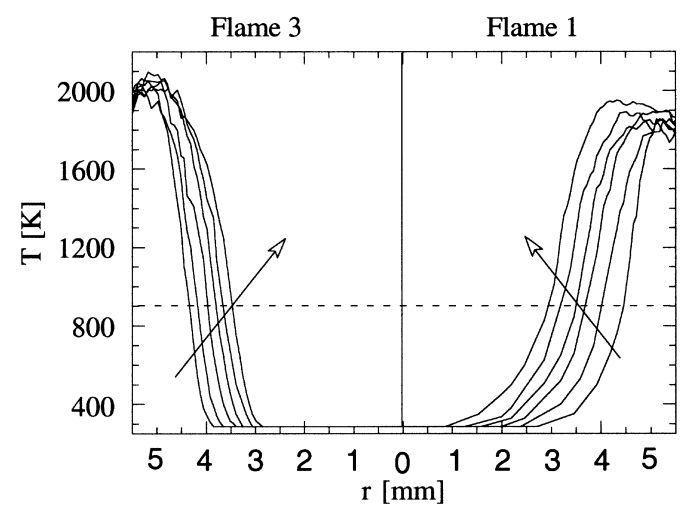

FIG. 5. Gaseous temperature as a function of the radial coordinate. Left section: flame 3 (at $z=1,4,8,12,16$, $20 \mathrm{~mm}$ ). Right section: flame 1 (at $z=1,4,8,12,16$, $20 \mathrm{~mm})$.

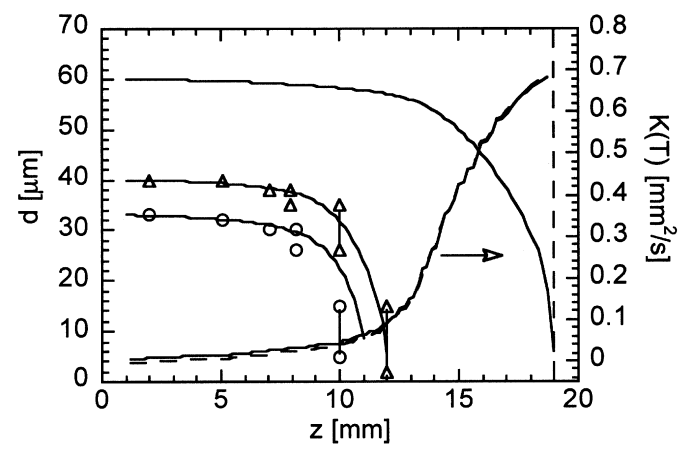

FIG. 6. Left ordinate: droplet size history as a function of the vertical coordinate for three different initial sizes: 33, 40, and $70 \mu \mathrm{m}$. Continuous lines are computed; open and black circles are the experimental data. Right ordinate: evaporation coefficient for the $70 \mu \mathrm{m}$ droplet based on different assumptions for the droplet wall temperature.

flame. (Note: Because of the small slip between gas and liquid phase, the droplet Reynolds number based on the slip velocity is on the order of $10^{-1}$, and the convective correction terms can be disregarded.)

In Fig. 5, we show the measured gas temperature for the two flames (flame 1 on the right, flame 3 on the left). For both flames, the peak temperature remains almost constant at different heights. Flame 3 has higher values because pressure effects reduce molecular dissociation. Thin-filament pyrometry was applicable only above $900 \mathrm{~K}$. The cold core temperature was measured to $288 \mathrm{~K}$ by a thermocouple. In the remaining region, we interpolated the temperature from $900 \mathrm{~K}$ to $288 \mathrm{~K}$ with a Stineman function [19], smoothly matching the slope in the hightemperature region to the zero gradient in the cold core.
Figure 6 shows the evolution of droplet diameter (left ordinate) along the droplet trajectory for three different droplets of initial sizes, 33, 40 , and $70 \mu \mathrm{m}$, respectively, in the atmospheric pressure flame. The position along each droplet trajectory is tracked by the axial coordinate in the abscissa of Fig. 6. Each droplet follows a different trajectory although the starting position $(r=3.00 \mathrm{~mm}$ and $z=1.00 \mathrm{~mm})$ is the same in all three cases. The calculated size evolution follows closely the experimentally measured one for both cases for which experiments were available. We can therefore assume that the underlying phenomenology is properly captured and can compute the size evolution of droplets of arbitrary size, as for the $70 \mu \mathrm{m}$ droplet for which no experimental data are available since no discernible peak in the distribution of Fig. 2 could be tracked at that size. In Fig. 6, the vertical dashed line at $z=19 \mathrm{~mm}$ represents the axial coordinate of the flame location where droplets with a starting size of $70 \mu \mathrm{m} \mathrm{com-}$ pletely vaporize as they reach the flame. Since the differences in trajectories between different droplets are relatively small, we can conclude that droplets smaller than $70 \mu \mathrm{m}$ with the same initial position will not reach the flame, while droplets of larger sizes will survive the reacting layer.

In Fig. 6 (right ordinate), the calculated vaporization constant for a $70 \mu \mathrm{m}$ droplet is also reported. The dashed line, represents $K(T)$ with $T_{\mathrm{w}}$ constant and equal to the ethanol boiling point of $351 \mathrm{~K}$, while the continuous line pertains to the case of the measured $T_{\mathrm{w}}=288 \mathrm{~K}$. For small values of $K$, corresponding to low-temperature values, the continuous line seems to be the more appropriate. Because of the temperature effect on the enthalpy of vaporization, the two curves coincide at a temperature that is around $1000 \mathrm{~K}$. At high values of $K$, the droplet wall temperature is known to be very close to the boiling point. So, the dashed line is the more appropriate for this region. Thus, it was assumed $T_{\mathrm{w}}=$ $288 \mathrm{~K}$ for $T_{\infty} \leq 600 \mathrm{~K}$, and $T_{\mathrm{w}}=T_{\mathrm{b}}=351 \mathrm{~K}$ for $T_{\infty}>600 \mathrm{~K}$.

Following Greenberg [5], we can define a vaporization Damköhler number defined as the ratio between residence time in the vaporizing part of the flame and vaporization time, $D a_{\mathrm{v}}=\left(\bar{K} / d_{0}^{2}\right)(s / \bar{v})$, where $s$ is the radial width of the vaporizing region and $\bar{v}$ is an average radial velocity. Droplets with $D a_{\mathrm{v}}$ $>1$, like the $33 \mu \mathrm{m}$ and $40 \mu \mathrm{m}$, will completely vaporize inside the flame, while droplets for which $D a_{\mathrm{v}}$ $<1$ will survive the diffusion flame and burn isolated on the outside. The case of $D a_{\mathrm{v}}=1$ corresponds to the $70 \mu \mathrm{m}$ droplet of Fig. 6 .

Figure 7 reports conditions of $D a_{\mathrm{v}}=1$ for different initial radial positions. The right half refers to flame 1 , the left half to flame 3 . The complete droplet trajectory for the $60 \mu \mathrm{m}$ droplet of Fig. 6 , starting at $r=3.00 \mathrm{~mm}$, is shown in the right section. The trajectory ends at the flame, here represented by the 
Flame 3

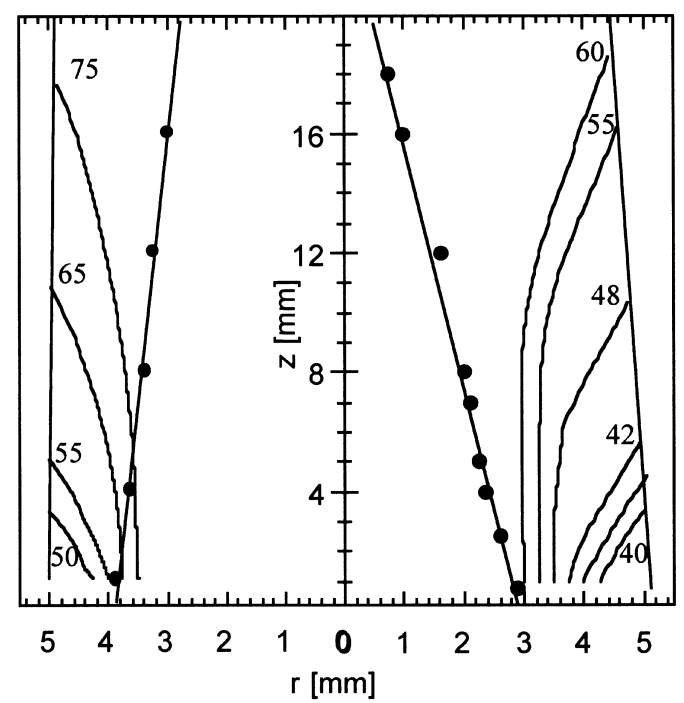

FIG. 7. Trajectories of the $D a=1$ droplets for the two flames. The left section pertains to flame 3, the right section to flame 1. Numbers on individual trajectories are the initial size of the droplets in micrometers. Also shown in each half of the figure are two oblique lines: the outermost corresponds to the flame position, the innermost to the edge of the cold region.

oblique line on the right side of the figure. The numbers on the trajectories represent the initial "coldcore" size of the droplets with unity $D a_{\mathrm{v}}$. The oblique line near the center of the figure represents the boundary of the cold core.

The critical diameter can be determined as $d_{\text {crit }}=$ $(\bar{K} s / \bar{v})^{0.5}$. If we assume that all the droplets that reach the flame have an equal average vaporization constant since they have a similar temperature history, the critical diameter will be a function only of the residence time in the flame. Droplets exiting the duct near the wall have a smaller path to the flame, which implies low residence time in the vaporization region and small critical diameters. Vice versa, droplets that exit the duct closer to the center have a longer vaporization time because of the large pathway to the flame. As a result, the critical diameters are larger. By the same token, for given trajectories corresponding to a critical diameter, if a droplet of that diameter exits the fuel tube at a large radial position it will punch through the flame.

In the left section of the same figure, trajectory and size of droplets with unity $D a_{\mathrm{v}}$ are presented for the flame at $3 \mathrm{~atm}$. As previously remarked, because of Peclet number effects, the cold region is broader than in flame 1. Notice that, for the same axial position, critical diameters are larger than for flame 1. Since the path to the flame is shorter, one would
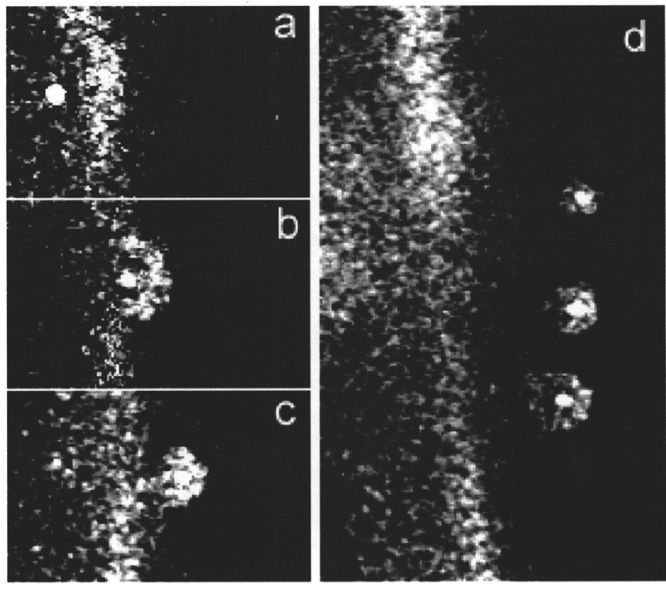

FIG. 8. Images of flame-droplet interaction. (a) Droplet approaching the flame; (b, c) Droplet crossing the flame position; (d) superimposed image at three different times of the same droplet burning outside the primary diffusion flame.

expect lower values of the critical diameter. Two effects combine to counteract the decrease in path length. First, the average vaporization constant increases because of the increased maximum temperature; second, the average radial velocity is lower in the high-pressure case, as shown in Fig. 3.

The results in Fig. 7 are consistent with the external appearance of the flames. At lower axial positions, critical diameters are smaller and many droplets of the initial distribution survive the diffusion flame eventually igniting on the outside. A comparison of the critical diameters with the initial size distribution shows that some droplets will eventually reach the diffusion flame also at a position relatively far away from the duct. These droplets do not always ignite outside of the reacting layer since their diameter is greatly reduced with respect to the initial value and might be below the critical value for ignition. Moreover, the thickness of the region of low oxidizer concentration outside the reacting layer increases with height, making the ignition process more difficult.

As a droplet approaches the flame from the fuel side and passes through, if conditions are favorable, it ignites, burns, and eventually extinguishes. Fig. 8 captures this sequence. It shows a series of photographs of droplets traveling left to right and illuminated by a He-Ne laser sheet collinear with the droplet trajectory. Together with the laser scattering, chemiluminescence by the reacting layer is shown. The nearly vertical, rippled chemiluminescent streak denotes the primary diffusion flame enveloping the vaporizing cloud. The fuel side is on the left of each 
picture. Fig. 8a shows the droplet approaching the flame. In view of the small slip, the flow can be considered approximately quiescent from the droplet vantage point. Thus, the local fuel mass fraction distribution around the droplet approaches that of a quiescent droplet vaporizing in a fuel-rich atmosphere. The position of the primary diffusion flame, at "infinity", is determined by the local gaseous fuel mass fraction gradients. At the droplet scale, because of the vaporization, the gas is locally enriched. The primary flame is displaced by the increased local concentration of fuel and a ripple develops (Fig. 8b). Subsequently (Fig. 8c), the droplet crosses the primary flame position and the local fuel concentration decreases. The oxidizer now penetrates the wake of the droplet, bringing the primary diffusion flame back to its original position, while the droplet escapes with a surrounding flame. Fig. $8 d$ shows three consecutive images of the same droplet burning with a surrounding flame. The flame shrinks while the droplet diameter decreases, and it will eventually extinguish when the droplets reaches a critical diameter. This ignition by a "flame transfer" process is probably unique of conditions of small slip between the two phases

\section{Conclusions}

Highly diluted spray diffusion flames were successfully stabilized at 1 and 3 atm in a coflow configuration. The structure of these flames reveals a cold core in which little or no vaporization occurs and a Peclet-dependent thermal boundary layer originating at the primary diffusion flame enveloping most of the spray. Most of the vaporization occurs in this boundary layer. At pressures above the atmospheric, because of density effects on thermal diffusivity, the growth of this layer above the burner is reduced and so is the vaporization region. Complete evaporation of the droplets before they reach the primary diffusion flame is ensured if a suitably defined Damköhler number of evaporation, $D a_{\mathrm{v}}$, is less than 1 . Conversely, if $D a_{\mathrm{v}}>1$, droplets penetrate the flame, ignite by a flame transfer process, and burn isolated on the oxidizer side. These conditions of internal or partial group combustion prevail in the lower part of the flame. This regime progressively shifts toward external group combustion, in which no direct droplet/flame interaction occurs, farther up in the flame.
Acknowledgments

The support of NSF Grant CTS-9904296 (Dr. Farley Fisher, Contract Monitor) is gratefully acknowledged.

\section{REFERENCES}

1. Chen, G., and Gomez, A., Proc. Combust. Inst. 24:1531-1539 (1992).

2. Li, S. C., Libby, P. A., and Williams, F. A., Proc. Combust. Inst. 24:1503 (1992).

3. Gao, L. P., D’Angelo, Y., Silverman, I., Gomez, A., and Smooke M. D., Proc. Combust. Inst. 26:1739 (1996).

4. Santoro, V. S., Kyritsis, D. C., and Gomez, A., Proc. Combust. Inst. 28 (2000).

5. Greenberg, J. B., Combust. Sci. Technol. 75:13 (1991).

6. Gomez, A., and Chen, G., in Physical and Chemical Aspects in Combustion-A Tribute to I. Glassman (F. L. Dryer and R. F. Sawyer, eds.), Gordon and Breach, Amsterdam, The Netherlands, 1997, p. 461.

7. Mao, C.-P. Wakamatsu, Y., and Faeth G. M., Proc. Combust. Inst. 18:337 (1980).

8. Russo, S., and Gomez, A., Combust. Flame 130:215 (2002).

9. Mulholland, J. A., Srivastava, R. K., Wendt, J. O. L., Agrawal, S. R., and Lanier, W. S., Combust. Flame 86:297 (1991).

10. Russo, S., "Experimental Study of Laminar Spray Flames at Moderately High Pressures," Ph.D. thesis, Department of Mechanical Engineering, Yale University, New Haven, CT, 2002.

11. Karpetis, A. N., and Gomez, A., J. Fluid Mech. 397:231 (1999).

12. Gañán-Calvo, A. M., Phys. Rev. Lett. 80(2):285 (1998).

13. Vilimpoc, V., and Goss, L. P., Proc. Combust. Inst. 22:1907 (1988).

14. Rosner, D. E., Transport Processes in Chemically Reacting Flow Systems, Butterworth-Heinemann, Boston, 1986

15. Davis, R. W., Moore, E. F., Roquemore, W. M., Chen, L.-D., Vilimpoc, V., and Goss, L. P., Combust. Flame 83:263 (1991).

16. Park, T. W., Aggarawal, S. K., and Katta, V. R. Combust. Flame 99:767 (1994).

17. Sugiyama, G., Proc. Combust. Inst. 25:601 (1994).

18. Gomez, A., and Glassman, I., Proc. Combust. Inst. 21:1087 (1986).

19. Synergy Software, Kaleidagraph Reference Guide, Reading, PA, 1996. 


\section{COMMENTS}

Eva Gutheil, University of Heidelberg, Germany. Did you perform measurements of droplet size distribution right at the nozzle exit to serve as input data for numerical computations? Is it possible to go beyond 0.9 MPa in pressure, or do you anticipate problems such as the experimental setup and diagnostic methods?

Author's Reply. We have detailed measurements of droplet size and velocity distributions $0.75 \mathrm{~mm}$ downstream of the nozzle exit, which could be used as input conditions of numerical models. Space limitations prevented us from showing the full data set, but we will be glad to make it available for modeling purposes.

As pressure increases, instabilities become more severe and will eventually prevent the establishment of a steady laminar flame. Diagnostically, a number of complications (e.g., beam steering, upper temperature limit of thin film pyrometry, soot formation) will arise, but most of them can be circumvented one way or another. As discussed in the oral presentation, the upper limit we could reach in the present experimental system was $0.9 \mathrm{MPa}$. Results on this flame and additional comparisons with the current ones will be reported in another article in preparation.

D. P. Mishra, Indian Institute of Technology, India. This simplified configuration can easily be modeled numerically. However, the use of non-monodisperse droplets will complicate simulation of the problem. Hence, I would have been delighted to see experimental data on monodisperse droplets. Did you measure the droplets at the inlet? What is the distribution of droplets?

Author's Reply. See reply to Guthiel, no additional reply necessary. 